Reprod. Nutr. Dévelop., 1983, 23 (2 B), 421-428.

\title{
Expression de la réponse glycogénique à l'insuline dans les hépatocytes fœtaux en culture
}

\author{
Christiane PLAS, Pierrette MENUELLE, Nadine FOREST, E. PRINGAULT \\ Laboratoire Interactions cellulaires, Université Paris 7, UER d'Odontologie, \\ Tour 43, 2 place Jussieu, 75221 Paris Cedex, France.
}

Summary. The expression of glycogenic response to insulin in cultured fetal hepatocytes.

In the present study, we used primary cultures of fetal rat hepatocytes which are highly suitable for studying the glycogenic effect of insulin and its regulation. After a lag in the period of culture in the presence of cortisol, glycogenic response to insulin developed together with a progressive accumulation of glycogen. When insulin was added, the rate of glycogen synthesis increased, becoming maximal after $2-3 \mathrm{~h}$, due to the activation of the glycogen synthase system already present. Modification of glycogen precursors in the medium did not alter the amplitude of the insulin effect. The glycogenic effect of insulin was unrelated to that of glucose load and occurred after the formation of glucose-1phosphate. This only happened when the cyclic AMP-dependent glycogenolytic system was not stimulated, since it was suppressed by low doses of glucagon. Insulin effect, which was time-dependent, ceased after $4 \mathrm{~h}$. This corresponded to a desensitization of hepatocytes without any alteration in the specific binding of insulin. These variations in the glycogenic effect of insulin were likely due to different causes; one of these could be the first events following the interaction of insulin with its receptor.

\section{Introduction.}

L'apparition des premières particules de glycogène dans I'hépatocyte se fait chez le foetus de rat au stade de 18 jours de gestation (Favard et Jost, 1966). La synthèse de glycogène $y$ est ensuite très active puisque juste avant la naissance (21 jours de gestation), la teneur en glycogène peut atteindre $10 \%$ du poids frais de l'organe. Les études in vivo ont montré que cette mise en réserve de glycogène implique l'intervention de facteurs hormonaux : glucocorticoïdes (Jacquot, 1959 ; Jost, 1966) mais aussi, à un moindre degré, insuline (Manns et Brockman, 1969 ; Rabain et Picon, 1974 ; Félix et Jacquot, 1976). Notre but est d'étudier l'effet direct de l'insuline sur la glycogenèse à l'aide d'hépatocytes fœtaux transplantés à des stades précédant l'accumulation de glycogène et maintenus en culture primaire dans des conditions permettant leur maturation fonctionnelle. 


\section{Matériel et méthodes.}

Les cultures primaires sont réalisées, à partir de foies de fœus de rat de 14 à 18 jours de gestation, selon la méthode de Plas, Chapeville et Jacquot (1973) permettant l'obtention d'une population homogène d'hépatocytes en monocouches. Le milieu de culture est le milieu NCTC 109 à $5,5 \mathrm{mM}$ glucose (milieu de culture "standard "), supplémenté ou non par $10 \%$ de sérum fœetal de veau. La glycogenèse et l'influence de l'insuline sur celle-ci sont étudiées par la mesure de la teneur en glycogène des cultures et la mise en œuvre d'expériences d'incorporation de glucose- ${ }^{14} \mathrm{C}$ dans le glycogène, ceci pendant des périodes courtes ( $30 \mathrm{~min}$ à $8 \mathrm{~h}$ ) ou plus longues. Ce dernier type de marquage permet d'estimer la valeur de la contribution du glucose du milieu à la formation du glycogène. Ces méthodes ont été précédemment décrites (Plas, Chapeville et Jacquot, 1973 ; Plas et al., 1979). Pour quantifier l'effet de l'insuline, nous utiliserons un index de stimulation défini par le rapport suivant : radioactivité incorporée dans le glycogène des cultures traitées par l'insuline/radioactivité incorporée dans le glycogène des cultures témoins. Le solvant de l'insuline et du glucagon est l'HCl $10 \mu \mathrm{M}$.

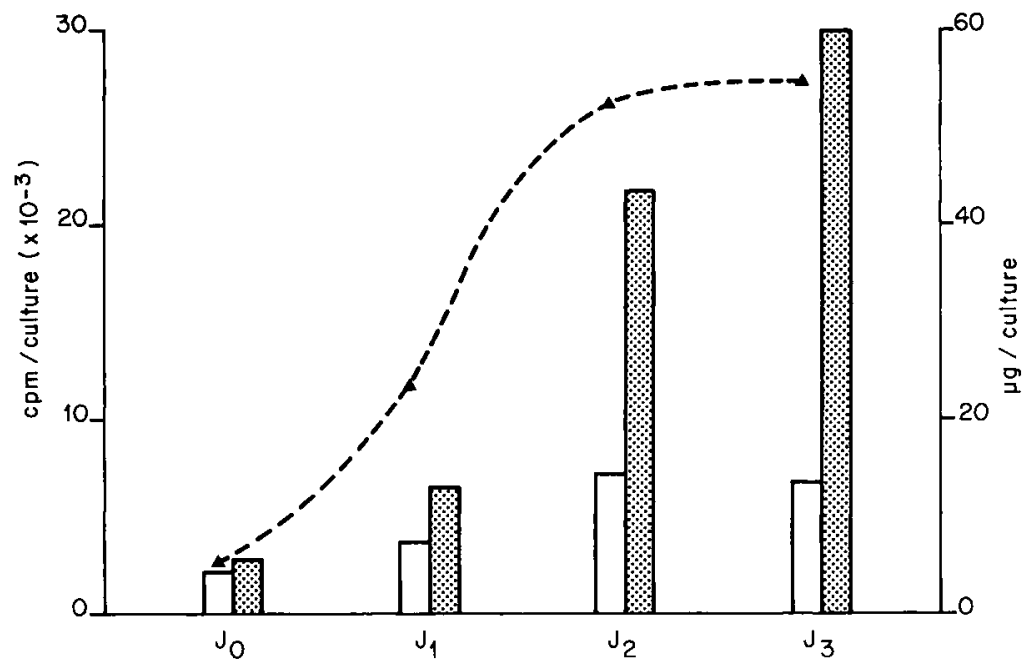

FIG. 1. - Développement de la réponse glycogénique à l'insuline et accumulation de glycogène. Après différents temps d'incubation en présence de cortisol $10 \mu \mathrm{M}\left\langle 6 \mathrm{~h}\right.$ à 3 jours, soit $\mathrm{J}_{0}$ à $J_{3}$ ), les cultures reçoivent le glucose ${ }^{14} \mathrm{C}(1 \mu \mathrm{Ci} / \mathrm{mg})$ ainsi que l'insuline $10 \mathrm{nM}$ ou le solvant de I'hormone. La radioactivité présente dans le glycogène est déterminée $4 \mathrm{~h}$ plus tard. Barres en pointillés: cultures ayant reçu l'insuline $10 \mathrm{nM}$; barres blanches : cultures témoins ; $(\mathbf{\Delta})$ : teneur en glycogène des cultures témoins. Cette expérience a été réalisée à l'aide d'hépatocytes de 17 jours.

\section{Résultats.}

Développement de la réponse glycogénique de l'insuline en culture. - Une réponse glycogénique à l'insuline ne peut être obtenue juste après la transplanta- 
tion des hépatocytes. Elle apparaît en culture corrélativement à l'augmentation progressive de la quantité de glycogène stocké. Chez les hépatocytes de 17 jours, l'index de stimulation relatif à l'effet de l'insuline sur l'incorporation de glucose- ${ }^{14} \mathrm{C}$ dans le glycogène au cours d'une période de $4 \mathrm{~h}$ est égal à $1,8,3,0$ et 4,4 au jour 1,2 et 3 de la culture alors que les teneurs en glycogène correspondantes sont 24,52 et $55 \mu \mathrm{g} /$ culture (fig. 1). L'étude comparée de cette maturation dans les hépatocytes prélevés à des stades différents montre un schéma qualitativement semblable. II faut noter cependant que, comme pour l'accumulation de glycogène (Plas, Chapeville, Jacquot, 1973), l'effet glycogénique de l'insuline s'exprime après un temps de culture plus important lorsque l'on examine des hépatocytes plus jeunes.

Le développement de la réponse glycogénique de l'insuline en culture nécessite des conditions de milieu définies qui permettent une maturation globale de la cellule, à la fois ultrastructurale et fonctionnelle. La présence de cortisol dans le milieu de culture s'est révélée un facteur important chez les hépatocytes fœetaux (Plas, Chapeville et Jacquot, 1973 ; Plas et Nunez, 1976) et chez les explants de foie fœtal (Eisen, Goldfine et Glinsmann, 1973). L'apparition de la réponse à I'insuline n'est pas liée à la mise en place du récepteur de l'hormone car une liaison spécifique de l'insuline aux hépatocytes est obtenue dès leur transplantation, même pour les stades les plus jeunes. L'une des hypothèses invoquées est que le cortisol provoquerait une augmentation de la glycogène synthase qui serait ensuite activée par l'insuline (Eisen, Goldfine et Glinsmann, 1973).
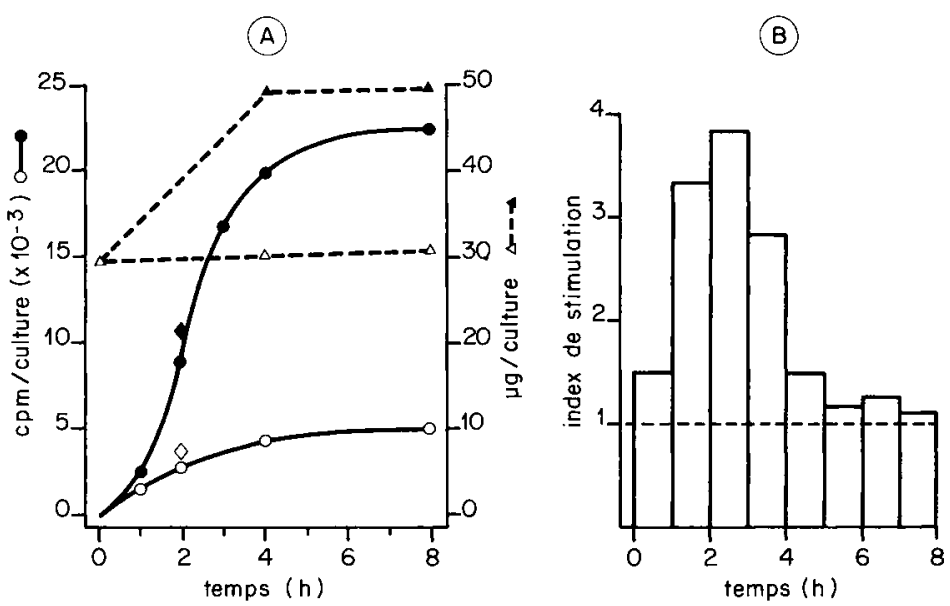

FIG. 2. - Décours temporel de l'effet glycogénique de l'insuline. (A) Au jour 3, les cultures reçoivent le glucose- ${ }^{14} \mathrm{C}(1 \mu \mathrm{Ci} / \mathrm{mg})$ ainsi que l'insuline $10 \mathrm{nM}$ ou son solvant; la cycloheximide $20 \mu \mathrm{M}$ est également ajoutée dans certaines cultures. Le glycogène $(\Delta, \Delta)$ et la radioactivité présente dans le glycogène $(\bullet, \bigcirc, \diamond, \diamond)$ sont déterminés pendant $8 \mathrm{~h}$. Cultures ayant reçu respectivement l'insuline $10 \mathrm{nM}(\boldsymbol{\Delta}, \bullet)$, le solvant $(\triangle, O)$, l'insuline $10 \mathrm{nM}$ et la cycloheximide $20 \mu \mathrm{M}(\bullet)$ et la cycloheximide $20 \mu \mathrm{M}(\diamond)$. (B) Des cultures menées parallèlement reçoivent également au jour 3 de l'insuline $10 \mathrm{nM}$ ou son solvant. Sont ensuite pratiquées des périodes successives d'incorporation de $1 \mathrm{~h}$ du glucose $-{ }^{14} \mathrm{C}(1 \mu \mathrm{Ci} / \mathrm{mg})$ dans le glycogène. En ordonnée est représenté l'index de stimulation de la glycogenèse par l'insuline (cf. Matériel et Méthodes). Cette expérience a été réalisée avec des hépatocytes de 18 jours. 
Effet glycogénique initial de l'insuline. - L'effet de l'insuline sur l'incorporation de glucose- ${ }^{14} \mathrm{C}$ dans le glycogène est significatif après $1 \mathrm{~h}$ d'action de l'hormone ; il est maximal après 2 à 3 h où un index de stimulation de 4 à 6 peut être obtenu. Après $4 \mathrm{~h}$, on observe une cessation de l'effet de l'insuline (fig. 2). L'effet glycogénique initial de l'insuline implique une augmentation de la vitesse de synthèse du glycogène (Plas et al., 1979). En effet, une inhibition de la glycogénolyse ne peut rendre compte de la stimulation observée, la demi-vie du glycogène dans l'hépatocyte fotal étant de 12-14 h (Plas, Chapeville et Jacquot, 1973 ; Plas et Nunez, 1975). De plus, la stimulation de la synthèse de glycogène par l'insuline, qui n'est pas modifiée par l'addition de cycloheximide à une dose inhibant $85 \%$ de la synthèse protéique, reflète l'activation d'enzymes préexistantes (fig. 2). Enfin, l'effet glycogénique initial de l'insuline est obtenu pour des concentrations physiologiques de l'hormone; la dose produisant un effet demimaximal est 0,3 nM (Plas et al., 1979).

Effets comparés de l'insuline et d'une charge en glucose sur la glycogenèse. - Que l'insuline soit présente ou non, l'incorporation de glucose- ${ }^{14} \mathrm{C}$ dans le glycogène augmente progressivement lorsque la concentration en glucose du milieu varie de 2 à $20 \mathrm{mM}$. De plus, la stimulation de la glycogenèse par l'insuline présente une amplitude identique quelle que soit la concentration en glucose du milieu (fig. 3). Des résultats semblables ont été obtenus dans les hépatocytes de jeune rat en culture primaire (Schudt, 1979). Donc, glucose et insuline agissent en favorisant la synthèse de glycogène et leurs effets sont additifs.

(A)

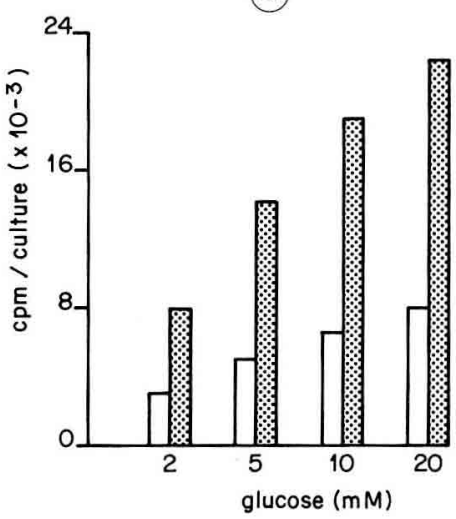

(B)

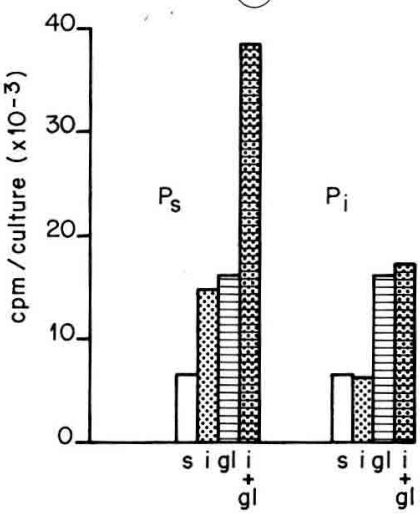

FIG. 3. - Effets du glucose et de l'insuline sur la glycogenèse. (A) Au jour 3, le milieu est prélevé et remplacé par un milieu à concentration variable de glucose $(2$ à $20 \mathrm{mM})$. Les cultures reçoivent alors le glucose $-{ }^{14} \mathrm{C}(1 \mu \mathrm{Ci} / \mathrm{mg})$ ainsi que l'insuline $10 \mathrm{nM}$ ou son solvant. La radioactivité présente dans le glycogène est déterminée $4 \mathrm{~h}$ plus tard. Barres pointillées : cultures avant reçu I'insuline $10 \mathrm{nM}$; barres blanches: cultures témoins. (B) Des cultures menées parallèlement reçoivent au jour 3 l'insuline $10 \mathrm{nM}$ (série $P_{\mathrm{i}}$ ) ou le solvant de l'hormone (série $P_{\mathrm{s}}$ ). Après $4 \mathrm{~h}$ de préincubation, les deux séries recoivent le glucose $-{ }^{14} \mathrm{C}(1 \mu \mathrm{Ci} / \mathrm{mg})$ en même temps que le solvant (s), l'insuline $10 \mathrm{nM}$ (i), le glucose $15 \mathrm{mM}$ (gl) ou l'insuline $10 \mathrm{nM}$ et le glucose $15 \mathrm{mM}(\mathrm{i}+\mathrm{gl}$ ). La radioactivité présente dans le glycogène est déterminée $4 \mathrm{~h}$ plus tard. Cette expérience a été réalisée avec des hépatocytes de 18 jours. 
La contribution du glucose du milieu à la formation du glycogène a été évaluée par comparaison de la radioactivité spécifique des unités-glucose dans le milieu et dans le glycogène (tabl. 1). Dans les conditions de milieu standard à $5,5 \mathrm{mM}$ glucose, la proportion de glucose participant à la formation du glycogène est de $60 \%$. En présence d'une charge de glucose, elle est augmentée et peut atteindre $80 \%$. En outre, la présence $d^{\prime}$ insuline entraîne un faible accroissement de cette contribution. II ressort de ces résultats qu'une partie importante du glycogène est synthétisée à partir du glucose-1-phosphate néoformé.

\section{TABLEAU 1}

Effets des substrats sur la contribution du glucose à la glycogenèse basale et stimulée par l'insuline

\begin{tabular}{|c|c|c|c|c|c|}
\hline Substrat & Addition & $\begin{array}{r}\text { Glyc } \\
\mathrm{cpm} / \text { culture }\end{array}$ & $\begin{array}{l}\text { yène } \\
\mu \mathrm{g} / \text { culture }\end{array}$ & $a / b$ & Index de stimulation \\
\hline $5 \mathrm{mM}$ glucose & $10 \mathrm{nM}$ insuline & $\begin{array}{l}47220 \\
83950\end{array}$ & $\begin{array}{l}45 \\
75\end{array}$ & $\begin{array}{l}0,60 \\
0,64\end{array}$ & $1,78 / 1,67$ \\
\hline $\begin{array}{l}5 \mathrm{mM} \text { glucose } \\
+2 \mathrm{mM} \text { fructose }\end{array}$ & $10 \mathrm{nM}$ insuline & $\begin{array}{ll}37 & 020 \\
66 & 260\end{array}$ & $\begin{array}{l}41 \\
69\end{array}$ & $\begin{array}{l}0,52 \\
0,55\end{array}$ & $1,79 / 1,68$ \\
\hline $\begin{array}{l}5 \mathrm{mM} \text { glucose } \\
+2 \mathrm{mM} \text { galactose }\end{array}$ & $10 \mathrm{nM}$ insuline & $\begin{array}{l}27370 \\
46830\end{array}$ & $\begin{array}{l}63 \\
96\end{array}$ & $\begin{array}{l}0,25 \\
0,28\end{array}$ & $1,71 / 1,52$ \\
\hline $\begin{array}{l}5 \mathrm{mM} \text { glucose } \\
+2 \mathrm{mM} \text { glycérol }\end{array}$ & $10 \mathrm{nM}$ insuline & $\begin{array}{l}52610 \\
88120\end{array}$ & $\begin{array}{l}51 \\
83\end{array}$ & $\begin{array}{l}0,59 \\
0,61\end{array}$ & $1,67 / 1,63$ \\
\hline $\begin{array}{l}5 \mathrm{mM} \text { glucose } \\
+2 \mathrm{mM} \text { lactate/pyruvate }\end{array}$ & $10 \mathrm{nM}$ insuline & $\begin{array}{r}64900 \\
109570\end{array}$ & $\begin{array}{l}54 \\
89\end{array}$ & $\begin{array}{l}0,69 \\
0,70\end{array}$ & $1,69 / 1,65$ \\
\hline $20 \mathrm{mM}$ glucose & $10 \mathrm{nM}$ insuline & $\begin{array}{r}99720 \\
175700\end{array}$ & $\begin{array}{r}81 \\
135\end{array}$ & $\begin{array}{l}0,70 \\
0,74\end{array}$ & $1,76 / 1,67$ \\
\hline
\end{tabular}

Le glucose- ${ }^{14} \mathrm{C}(1 \mu \mathrm{Ci} / \mathrm{mg})$ est introduit dans le milieu dès le début de la culture et maintenu pendant toute la durée de l'expérience. Au jour 1, le milieu est prélevé et remplacé par un milieu standard ( $5 \mathrm{mM}$ glucose) supplémenté comme indiqué dans le tableau. Les cultures reçoivent alors l'insuline $10 \mathrm{nM}$ ou son solvant. Le glycogène et la radioactivité présente dans le glycogène sont déterminés $30 \mathrm{~h}$ plus tard. $\mathrm{a} / \mathrm{b}$ : rapport entre la radioactivité spécifique des unités glucose dans le glycogène (a) et celle du glucose dans le milieu (b). Cette expérience a été réalisée à l'aide d'hépatocytes de 18 jours.

Influence de substrats gluconéogéniques sur l'effet de l'insuline. - L'effet de l'insuline a été étudié après modification du milieu de culture standard par une supplémentation en précurseurs présumés du glycogène : fructose, galactose, glycérol et lactate-pyruvate. Tous ces substrats participent au métabolisme énergétique de l'hépatocyte car une économie du glucose consommé dans le milieu est observée en leur présence. La contribution du glucose à la formation du glycogène varie en fonction du substrat ajouté ; en particulier, elle est diminuée en présence de fructose et de galactose (tabl. 1). L'addition d'insuline provoque toujours un léger accroissement de cette contribution. Aussi l'amplitude de l'effet glycogénique de l'insuline est identique quel que soit le substrat utilisé. Ces résultats montrent que l'insuline n'interfère pas avec les voies métaboliques conduisant à la formation du glucose-1-phosphate et que son action s'exerce en bout de chaîne métabolique. 
Désensibilisation des hépatocytes à l'insuline après exposition à l'hormone. - L'effet glycogénique initial de l'insuline cesse après $4 \mathrm{~h}$ de présence de l'hormone (fig. 2). Cet arrêt n'est pas dû à une inactivation de l'insuline car celle-ci est très faiblement dégradée dans le milieu de culture (Menuelle et Plas, 1981). Egalement une addition supplémentaire d'insuline n'a pas d'effet. En revanche, une charge de glucose provoque chez ces hépatocytes désensibilisés à l'insuline un effet glycogénique de grande amplitude (fig. 3) ; la cessation de l'effet de l'insuline n'est donc pas due à une saturation de la capacité de synthèse du glycogène de l'hépatocyte. Elle n'implique pas l'intervention du système antagoniste glycogénolytique car la vitesse de dégradation du glycogène ri'est pas modifiée : elle résulte d'un défaut dans l'activation de la voie de synthèse du glycogène par l'insuline. Cette cessation présente en outre un caractère transitoire puisqu'un effet glycogénique peut réapparaître après $12 \mathrm{~h}$ d'exposition continue à l'hormone (Plas et al., 1979).

La désensibilisation, totale après une exposition à l'insuline $10 \mathrm{nM}$, est un processus dépendant de la dose de l'hormone : le degré de désensibilisation visà-vis d'une seconde dose d'insuline maximale pour l'effet biologique est fonction de la première dose administrée (Plas et al., 1979). Le phénomène de diminution du nombre de récepteurs de l'insuline après exposition prolongée à l'hormone (« down regulation ») décrit dans les hépatocytes adultes en culture primaire (Blackard, Guzelian et Small, 1978 ; Caro et Amatruda, 1980) n'est pas détectable dans les hépatocytes fœtaux 6Menuelle et Plas, 1981). En présence de chloroquine qui modifie le devenir du complexe insuline-récepteur dans la cellule, on observe une perte spécifique de l'effet glycogénique de l'insuline sans modification de l'effet glycogénique d'une charge de glucose (Plas et Desbuquois, 1982). Cette drogue révèle donc une situation analogue à celle obtenue dans les hépatocytes désensibilisés à l'hormone. La désensibilisation à l'insuline, qui n'implique pas une modification de l'interaction primaire de l'insuline avec son récepteur, pourrait provenir d'un défaut dans le fonctionnement du récepteur. Celui-ci, par exemple, n'assurerait plus la production du "médiateur " de l'insuline, peptide activant la glycogène synthase phosphatase en milieu acellulaire (Larner et al., 1979). Cependant une altération dans une étape ultérieure de l'action de l'hormone ne peut être exclue.

Régulation glycogénolytique et effet glycogénique de l'insuline. - L'addition de glucagon induit une réponse glycogénolytique rapide qui est médiée par l'activation du système adénylate cyclase. La dose d'hormone nécessaire pour obtenir un effet demi-maximal est $0,1 \mathrm{nM}$ (Plas et Nunez, 1975). Lorsque I'on ajoute simultanément insuline et glucagon à une dose $10 \mathrm{nM}$, qui pour les deux hormones produit un effet maximal, seule la réponse glycogénolytique est exprimée (fig. 4). L'effet antagoniste du glucagon est déjà sensible pour une dose de $0,1 \mathrm{nM}$ qui annule totalement l'effet glycogénique d'une dose d'insuline $10 \mathrm{nM}$, ceci bien que les doses de glucagon et d'insuline produisant un effet demimaximal soient voisines. L'effet glycogénique de l'insuline est donc très sensible à l'activation du système adénylate cyclase par le glucagon.

$\mathrm{D}^{\prime}$ autre part, lorsque les hépatocytes ont été incubés pendant $4 \mathrm{~h}$ en présence de glucagon $10 \mathrm{nM}$, l'addition d'insuline produit alors une réponse glyco- 
génique de grande amplitude malgré la présence de l'hormone glycogénolytique (fig. 4). En effet, l'exposition prolongée au glucagon a induit une désensibilisation spécifique vis-à-vis de cette hormone (Plas et Nunez, 1975). Le mécanisme de ce processus a pu être attribué à un blocage dans le couplage récepteur du glucagon-adénylate cyclase sans modification importante de la liaison spécifique de l'hormone (Plas et Nunez, 1975; Moncany et Plas, 1980). Comme dans le foie adulte (Exton et al., 1972), l'insuline peut contrecarrer l'effet de faibles concentrations de glucagon, mais l'hormone glycogénolytique apparaît dominante dans I'hépatocyte fœtal. La désensibilisation au glucagon rend néanmoins possible la réponse à l'insuline en présence de fortes concentrations de glucagon.

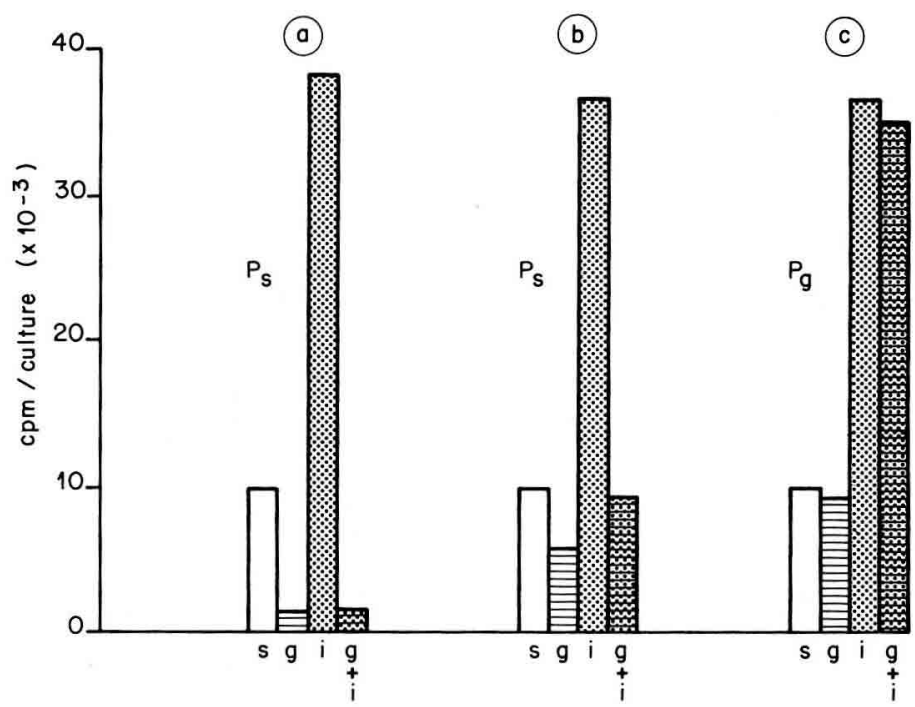

FIG. 4. - Effets du glucagon et de l'insuline sur la glycogenèse. Au jour 3, des cultures menées parallèlement sont préincubées en présence de glucagon $10 \mathrm{nM}$ (série $\mathrm{Pg}$ ) ou du solvant de l'hormone (séries Ps). Après $4 \mathrm{~h}$ d'incubation dans ces conditions, chaque série de cultures reçoit le glucose ${ }^{14} \mathrm{C}(1 \mu \mathrm{Ci} / \mathrm{mg})$ en même temps que le solvant $(\mathrm{s})$, le glucagon $(\mathrm{g}) 10 \mathrm{nM}$ (exps. a et c) ou $0,1 \mathrm{nM}$ (exp. b), I'insuline $10 \mathrm{nM}$ (i) ou bien le glucagon $10 \mathrm{nM}$ (exps. a et c) ou $0,1 \mathrm{nM}(\exp$. b) et l'insuline $10 \mathrm{nM}(\mathrm{g}+\mathrm{i})$. La radioactivité présente dans le glycogène est déterminée $4 \mathrm{~h}$ plus tard. Cette expérience a été réalisée avec des hépatocytes de 18 jours.

\section{Conclusion.}

Les hépatocytes fœetaux en culture primaire représentent un système cellulaire particulièrement sensible pour l'étude de l'effet glycogénique de l'insuline et de ses régulations. L'expression de cet effet ne s'exerce qu'à condition que le système antagoniste glycogénolytique AMP cyclique-dépendant ne soit pas stimulé. L'effet glycogénique de l'insuline est indépendant de l'effet d'une charge de glucose et intervient au-delà de la formation du glucose-1-phosphate. II implique une augmentation de la vitesse de synthèse du glycogène supposant l'activation du système glycogène synthase qui doit préexister. Des variations importantes de l'effet glycogénique se produisent en fonction du développement de 
I'hépatocyte, du temps de présence de l'hormone et de l'intervention du glucagon. Les causes qui les induisent sont vraisemblablement différentes, l'une d'entre elles pourrait être liée au fonctionnement du récepteur de l'insuline.

$8^{*}$ Réunion du groupe Développement I.N.R.A., Tours, 12-13 mai 1982.

Remerciements. - Ce travail a bénéficié des contrats INSERM ATP 74.79.106 et CNRS ATP 03.3824.

\section{Références}

BLACKARD W. G., GUZELIAN P. S., SMALL M. E., 1978. Down regulation of insulin receptors in primary cultures of adult rat hepatocytes in monolayer. Endocrinology, 103, 548-553.

CARO J. F., AMATRUDA J. M., 1980. Insulin receptors in hepatocytes: postreceptor events mediate down regulation. Science, 210, 1029-1031.

EISEN H. J., GOLDFINE I. D., GLINSMANN W. H., 1973. Regulation of hepatic glycogen synthesis during fetal development : roles of hydrocortisone, insulin and insulin receptors. Proc. nat. Acad. Sci. USA, 70, 3454-3457.

EXTON J. H., LEWIS S. B., HO R. J., PARK C. R., 1972. The role of cyclic AMP in the control of hepatic glucose production by glucagon and insulin. Adv. cyclic Nucleotides Res., 1, 91-101.

FAVARD P., JOST A., 1966. Différenciation et charge en glycogène de l'hépatocyte du fœtus de Rat normal ou décapité (étude en microscopie électronique). Arch. Anat. microsc. Morphol. exp., 55, 603-632.

FELIX J. M., JACQUOT R. L., 1976. Effects of sub-total gastro-intestinal pancreatectomy of the rat foetus. I. Endocrinol., 69, 77-83.

JACQUOT R., 1959. Recherches sur le contrôle endocrinien de l'accumulation de glycogène dans le foie chez le foetus de Rat. J. Physiol. Paris, 51, 655-721.

JOST A., 1966. Problems of fetal endocrinology: the adrenal glands. Rec. Progr. Horm. Res., 22, 541-574.

LARNER J., GALASKO G., CHENG K., De PAOLI-ROACH A. A., HUANG L., DAGGY P., KELLOGG J., 1979. Generation by insulin of a chemical mediator that controls protein phosphorylation and dephosphorylation. Science, 206, 1408-1410.

MANNS J. G., BROCKMAN R. P., 1969. The role of insulin in the synthesis of fetal glycogen. Can. J. Physiol. Pharmacol., 47, 917-921.

MENUELLE P., PLAS C., 1981. Relationship between insulin binding and glycogenesis in cultured fetal hepatocytes. Diabetologia, 20, 647-653.

MONCANY M. L. J., PLAS C., 1980. Interaction of glucagon and epinephrine in the regulation of adenosine $3^{\prime}, 5^{\prime}$-monophosphate-dependent glycogenolysis in the cultured fetal hepatocytes. Endocrinology, 107, 1667-1675.

PLAS C., CHAPEVILLE F., JACQUOT R., 1973. Development of glycogen storage ability under cortisol control in primary cultures of rat fetal hepatocytes. Develop. Biol., 32, 82-91.

PLAS C., DESBUQUOIS B., 1982. Receptor-mediated insulin degradation and insulin-stimulated glycogenesis in cultured foetal hepatocytes. Biochem. J., 202, 333-341.

Plas C., MENUElle P., MONCANY M. L. J., FUlChIGNONI-LATAUd M. C., 1979. Time dependence of the glycogenic effect of insulin in cultured fetal hepatocytes. Diabetes, 28. 705-712.

PLAS C., NUNEZ J., 1975. Glycogenolytic response to glucagon of cultured fetal hepatocytes. Refractoriness following prior exposure to glucagon. $/$. biol. Chem., 250, 5304-5311.

PLAS C., NUNEZ J., 1976. Role of cortisol on the glycogenolytic effect of glucagon and on the glycogenic response to insulin in fetal hepatocyte culture. J. biol. Chem., 251, 1431-1437.

RABAIN F., PICON L., 1974. Effect of insulin on the materno-fetal transter of glucose in the rat. Horm. Metab. Res., 6, 376-380.

SCHUDT C., 1979. Regulation of glycogen synthesis in rat-hepatocyte cultures by glucose, insulin and glucocorticoids. Eur. I. Biochem, 97, 155-160. 